\title{
2020 EULAR points to consider for the prevention, screening, assessment and management of non- adherence to treatment in people with rheumatic and musculoskeletal diseases for use in clinical practice
}

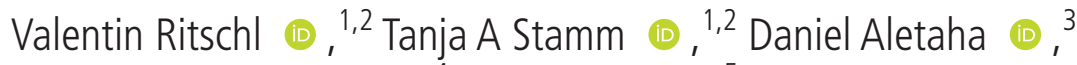 \\ Johannes W J Bijlsma (1) , ${ }^{4}$ Peter Böhm 다, ${ }^{5}$ Razvan Gabriel Dragoi (1), ${ }^{6}$

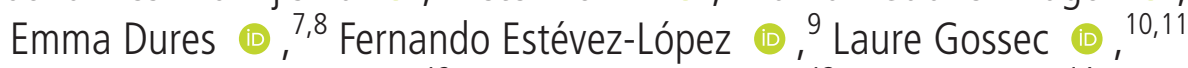 \\ Annamaria lagnocco $\bullet{ }^{12}$ Andrea Marques $\odot{ }^{1},{ }^{13}$ Ellen Moholt, ${ }^{14}$ Michal Nudel, ${ }^{15}$

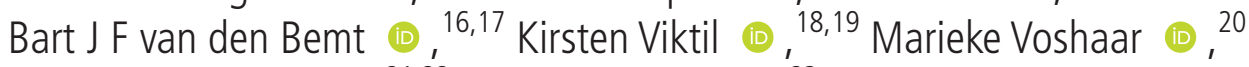 \\ Annette de Thurah (i), 21,22 Loreto Carmona (1) ${ }^{23}$
}

\begin{abstract}
Handling editor Désirée van der Heijde

- Additional material is published online only. To view, please visit the journal online (http://dx.doi.org/10.1136/ annrheumdis-2020-218986).

For numbered affiliations see end of article.
\end{abstract}

\section{Correspondence to}

Tanja A Stamm, Section for Outcomes Research, Medical University of Vienna, Wien, 1090 Wien, Austria;

tanja.stamm@meduniwien.ac.at

Received 27 August 2020 Revised 23 October 2020 Accepted 26 October 2020 Published Online First 18 December 2020

Check for updates

(C) Author(s) (or their employer(s)) 2021. No commercial re-use. See rights and permissions. Published by BMJ.

To cite: Ritschl V,

Stamm TA, Aletaha D,

et al. Ann Rheum Dis

2021:80:707-713

\section{ABSTRACT}

Background Non-adherence to treatment could preclude reaching an optimal outcome. Thirty to $80 \%$ of patients with rheumatic and musculoskeletal diseases (RMDs) do not adhere to the agreed treatment.

Objectives The objective was to establish points to consider (PtCs) for the prevention, screening, assessment and management of non-adherence to (non-) pharmacological treatments in people with RMDs. Methods An EULAR task force (TF) was established, and the EULAR standardised operating procedures for the development of PtCs were followed. The TF included healthcare providers (HCPs), comprising rheumatologists, nurses, pharmacists, psychologists, physiotherapists, occupational therapists and patient-representatives from 12 European countries. A review of systematic reviews was conducted in advance to support the TF in formulating the PtCs. The level of agreement among the TF was established by anonymous online voting.

Results Four overarching principles and nine PtCs were formulated. The PtCs reflect the phases of action on non-adherence. HCPs should assess and discuss adherence with patients on a regular basis and support patients to treatment adherence. As adherence is an agreed behaviour, the treatment has to be tailored to the patients' needs. The level of agreement ranged from 9.5 to 9.9 out of 10 .

Conclusions These PtCs can help HCPs to support people with RMDs to be more adherent to the agreed treatment plan. The basic scheme being prevent nonadherence by bonding with the patient and building trust, overcoming structural barriers, assessing in a blame-free environment and tailoring the solution to the problem

\section{INTRODUCTION}

Thirty to $80 \%$ of people with rheumatic and musculoskeletal diseases (RMDs) do not follow the recommended treatment plan. ${ }^{1-3}$ Non-adherence equally affect medication, non-pharmacological interventions and keeping follow-up appointments and are associated with worse outcomes, increased risk of cardiovascular disease, decreased functioning and loss of health-related quality of life. ${ }^{1-7}$ Strategies to reduce non-adherence are thus essential to achieve an optimal outcome. ${ }^{4-6}$

The problem of non-adherence is addressed in some EULAR recommendations on the management of specific health conditions or in the role of professionals, but none specifies interventions in or actual directions on how to improve non-adherent behaviour. ${ }^{8-11}$ All these recommendations focus on specific aspects of non-adherence and do not cover the multifaceted nature of this phenomenon, such as its detection or assessment.

Although general recommendations are lacking, a large number of studies have tested various interventions targeting non-adherence, including screening and assessment of non-adherence, ${ }^{1}$ provision of equitable and coordinated access to treatment through, for example, flexibility in scheduling or financial resources according to the respective health system, ${ }^{12}$ integration of patients in treatment decisions, ${ }^{12}$ enhancement of patients' autonomy, ${ }^{13}$ stratification and individualisation of interventions based on the needs and preferences of patients including psychosocial markers, ${ }^{8} 9$ information and education, ${ }^{10-13}$ systems to remind patients about appointments, intake of medication and exercises whenever necessary, ${ }^{14}$ compatibility of treatment interventions to the daily routines of patients, ${ }^{9} 13$ and offer opportunities to get in touch with other individuals with similar health conditions or other social support. ${ }^{12} 13$ Interventions are delivered by rheumatologists, other medical specialists, general practitioners or health professionals in rheumatology (HPRs) (ie, nurses, pharmacists, physiotherapists, occupational therapists and psychologists) in close collaboration with each other in primary and secondary care settings.

The objective of the present work is to establish points to consider (PtCs) for the prevention, screening, assessment and management of nonadherence in people with RMDs for use in daily clinical practice.

The users of these PtCs are intended to be rheumatologists and HPRs (together will be referred as 
healthcare providers or HCPs), patients and caregivers, regulators, trainers and others, at the individual or organisational level (eg, patient organisations, pharmaceutical and/or insurance companies). Regarding their scope, these PtCs are applicable to all RMDs, except those with an acute or subacute course (eg, some viral arthritis), as longer duration of diseases increase the chances of non-adherence. ${ }^{4}$ In addition, the scope of the PtCs does not include children and adolescents, as their non-adherent behaviour differs from that of adults, mainly on its great reliance on social support of caregivers. ${ }^{15}$ We acknowledge that different RMDs may have specific problems-for example, nonsymptomatic conditions, such as osteoporosis, pose additional challenges to motivate a patient to follow a long-term prescription (prescription in this context refers to any instruction (mostly written) from a physician or health professional in rheumatology stating the form, dosage and kind of treatment, including but not limited to medications, exercises, diets and follow-up appointments); however, non-adherence affects them all, and only very exceptionally a PtC for an RMD might not apply to another.

In addition, these PtCs only refer to non-adherence to pharmacological or non-pharmacological treatments that are prescribed or recommended. They are not including non-adherence to lifestyle changes, such as diet, weight loss and smoking or to visit schedules. Regarding medication, the task force decided that symptomatic medicines may not be the specific objective of these PtCs, and that non-pharmacological treatments should be restricted to exercises and medical devices (eg, splints). Exercises may be defined as a type of physical activity that is planned, structured and purposeful. ${ }^{16} 17$

Finally, in the context of these PtCs and following the definition of the WHO, non-adherence is defined as the extent to which a person's behaviour does not correspond with the agreed prescription, of pharmacological or non-pharmacological treatments, by an HCP. ${ }^{4}$ Besides being intentional or non-intentional, non-adherence (1) may occur at the start of treatment (initial non-acceptance), and so the patient never collects the prescription, or does not sign up to exercises, and hence does not follow any of the prescription; (2) may be a result of a poor execution, either by taking an incorrect dose, taking the drug at a wrong time or by decreasing or increasing the frequency of doses (or their equivalents in exercises or the use of medical devices) or (3) may be due to discontinuation of the treatment at any time during the treatment course. ${ }^{1}$

\section{METHODS}

These PtCs were developed according to the consensus process suggested by the EULAR Standard Operating Procedures. ${ }^{18}$ An international expert task force was established by a steering committee (LC, VR, AdT and TAS), and included people with RMDs $(n=2)$, EMEUNET members $(n=3$ (AM, RGD and $\mathrm{VR})$ ), and representatives from relevant HCP groups: nurses $(n=3)$, occupational therapists $(n=2)$, psychologists $(n=3)$, physiotherapists $(n=1)$, pharmacists $(n=2)$ and rheumatologists $(n=6)$, all of whom had various levels of expertise in the field of non-adherence and came from a broad geographical distribution across Europe. A systematic review (SR) of reviews and meta-analysis on existing strategies to prevent or mitigate nonadherence, supervised by the methodologist and the convenors, was presented at a first task force meeting (the SR is subject of a separate publication ${ }^{19}$ ). In this meeting, the scope, users, structure of the document and overarching principles were established by nominal group technique, as well as additional clinical questions to be addressed by SR. These clinical questions were converted into Population Intervention Comparison Outcome questions by the convenors (LC and VR), methodologist (AdT) and research fellows (VR and JBNT), and the search strategies developed, by an experienced Librarian, in Medline, CINAHL, web of science, science direct and the Cochrane Database of SR. The quality of the selected reviews was assessed using 'A MeaSurement Tool to Assess systematic Reviews' (available at https://amstar.ca/). The quality and risk of bias of the original studies were obtained directly from the published reviews (ie, Cochranes Risk of Bias tool $^{20}$ for intervention studies and QUADAS- $2^{21}$ for assessment studies). Because of the high heterogeneity, the evidence was synthesised qualitatively. In a second meeting of the task force, the results of the SR were discussed and the PtCs were formulated. Data from the SR were categorised according to the Oxford system for levels of evidence, ${ }^{22}$ and statements were voted and discussed using a three round Delphi technique. Level of agreement (LoA)/voting was scored anonymously (www.sli. do) on a numerical rating scale ranging from zero (completely disagree) to 10 (completely agree). The first two Delphi rounds were performed during the second task force meeting. Agreement in the Delphi was defined as $>80 \%$ of experts within the task force voting in favour (nine or 10) or against (one or two) an item. Items with agreement against were excluded from the list. Items with agreement in favour were maintained without further voting, unless reformulation was proposed. All intermediate items and those that needed reformulation were voted in a second round. Finally, in the third Delphi round (done by electronic communication (www.surveymonkey.de)), task force members were asked to give their final rating on every point to consider. All members of the task force were asked to respond during each round.

\section{RESULTS}

The results of the taskforce efforts are divided into four overarching principles and nine PtCs. The difference, in absence of strong evidence, is that overarching principles are not suggestions on what to do, but more principles to understand why and how the following points are formulated. They are explained and justified in detail below and presented in table 1 with the accompanying level of evidence (LoE), grade of recommendation (GR) and LoA. ${ }^{22}$

\section{Overarching principles \\ Overarching principle A: adherence impacts the outcomes of people with RMDs}

In RMDs, non-adherence has been associated with worse disease severity, increased pain and fatigue, higher rates of depression, lower function and a decrease in quality of life and physical activity. $^{19}$

\section{Overarching principle B: shared decision making is key, since} adherence is a behaviour following an agreed prescription

Ideally, patients and their HCPs should agree on the recommended treatment, including duration, dosage and frequency of medication intake, or exercises or device use over a period of time. To make an informed decision, patients need to understand their choices (ie, shared decision making, or SDM). The SDM process has been defined as 'an approach where HCPs and patients share the best available evidence when faced with the task of making decisions, and where patients are supported to consider options, to achieve informed preferences'. ${ }^{23}$ During the SDM process, mutual expectations in terms of efficacy, safety and monitoring should be discussed, as well as fears and 
Table 1 Overarching principles and points to consider for the prevention, screening, assessment and management of non-adherence in people with RMDs for use in the daily clinical practice

\begin{tabular}{|c|c|c|c|c|}
\hline \multicolumn{5}{|c|}{ Adherence is defined as the extent to which a person's behaviour corresponds with the agreed prescription ${ }^{* 4}$} \\
\hline \multicolumn{4}{|c|}{ Overarching principles } & \multirow{2}{*}{$\begin{array}{l}\text { LoA } \\
98.6\end{array}$} \\
\hline A & Adherence impacts the outcomes of people with RMDs. & & & \\
\hline B & Shared decision making is key, since adherence is a behaviour following an agreed prescription. & & & 95.8 \\
\hline C & Adherence is influenced by multiple factors. & & & 97.5 \\
\hline D & Adherence is a dynamic process that requires continuous evaluation. & & & 96.2 \\
\hline \multicolumn{2}{|c|}{ Points to consider } & LoE & GR & LoA \\
\hline 1 & All HCPs involved in the management of people with RMDs should take responsibility for promoting adherence. & 5 & $\mathrm{D}$ & 98.6 \\
\hline 2 & Effective patient-health professional communication should be applied to enhance adherence. & 5 & $\mathrm{D}$ & 99.4 \\
\hline 3 & Barriers and facilitators of adherence of a specific patient to a specific prescription should be appropriately evaluated. & 5 & $\mathrm{D}$ & 95.2 \\
\hline 4 & Patient education should be provided for people with RMDs as an integral part of standard care. & $1 \mathrm{~A}$ & A & 95.9 \\
\hline 5 & Care should be tailored to patient preferences and goals to enhance adherence. & 5 & $\mathrm{D}$ & 98.4 \\
\hline 6 & Adherence should be discussed regularly based on open questions and particularly when disease is not well controlled. & 5 & D & 98.9 \\
\hline 7 & $\begin{array}{l}\text { The HCP should explore which factors might negatively influence adherence, including: opportunity (eg, availability or cost), capability (eg, memory } \\
\text { problems), motivation (eg, concerns). }\end{array}$ & 5 & D & 93.8 \\
\hline 8 & $\begin{array}{l}\text { Together with the patient, the HCP should tailor the approach to overcome individual barriers to adherence, for example, simplifying the regimen, using } \\
\text { reminders, providing education, discussing the patient's beliefs on treatments. }\end{array}$ & 5 & D & 97.9 \\
\hline 9 & When specific expertise or interventions for adherence are needed, they should be made available to patients. & 5 & $\mathrm{D}$ & 97.7 \\
\hline
\end{tabular}

*Prescription in this context refers to any instruction (mostly written) from a physician or health professional in rheumatology stating the form, dosage and kind of treatment, including but not limited to medications, exercises, diets and follow-up appointments.

$\mathrm{GR}$, grade of recommendation ${ }^{20}$; HCP, healthcare providers; LoA, Level of agreement of task force members on a numeric rating scale from 0 (no agreement) to 10 (perfect agreement); LoE, level of evidence; RMDs, rheumatic and musculoskeletal diseases.

necessities about the specific treatment. The patient should be informed about the need to be adherent and how adherence will be evaluated. Agreement is part of the definition of adherence in the sense that if the patient did not agree to start with, it could not be considered non-adherence. Although, SDM is ambitious in systems with time constraints and low availability of professionals, it cannot be overlooked if we want to action at all on non-adherence.

Overarching principle $\mathrm{C}$ : adherence is influenced by multiple factors Patients may have comorbidities, multiple treatments, circumstances, cognitions and preferences that need to be taken into account, when addressing non-adherence. Health systems are complex and, in each setting, different HCPs may have different roles. These PtCs have an integrative approach that goes beyond the clinical encounter, as they stress that all, HCPs and patients, are involved in the care of patients.

Overarching principle $\mathrm{D}$ : adherence is a dynamic process that requires continuous evaluation

Patients' beliefs and fears about prescribed treatments, as well as behaviours, are influenced by experience and by external and internal determinants, for example, depression, other comorbidities, side effects, lack of efficacy or life changes that pose logistic hurdles. We cannot assume that a person will continue to be adherent or non-adherent to any given treatment, and that this will be maintained and stable across prescriptions.

\section{Points to consider}

PtC 1: all HCPs involved in the management of people with RMDs should take responsibility for promoting adherence

Effective interventions to reduce non-adherence in RMDs were reviewed by our group, and we confirmed that HCP, both physicians-namely, rheumatologists, GP and orthopaedic surgeonsand HPRs-such as nurses, physiotherapists, occupational therapists, psychologists, exercise physiologists, patient educators and pharmacists-were involved in the delivery of these interventions. ${ }^{19}$ Nieuwlaat et al underscore in their Cochrane review that nurses, pharmacists and therapists are increasingly becoming part of delivering interventions that target nonadherent behaviour in people with RMDs. ${ }^{24}$ This $\mathrm{PtC}$ gives responsibility to the full care team. It highlights both the relevant role of these HPRs and also both the need to acknowledge their efforts, and to coordinate teams beyond the rheumatology clinic. Training in specific interventions-for example, motivational interview or communication skills-may also be behind effective or non-effective interventions. ${ }^{25}$ Very importantly, the HCPs should be involved and should be trained as well.

\section{PtC 2: effective patient-health professional communication should} be applied to enhance adherence

An overview of SR on strategies proven effective to reduce nonadherence to medication and prescribed exercises found that effective communication was part of most multifaceted interventions proven successful. Unfortunately, the content and nature of effective communication is not well detailed. ${ }^{19}$ Despite the absence of consensus on a definition of effective communication, its components-namely, empathy, open questions or bilateral feedback, among others-were discussed and detailed by the task force (see online supplemental table S3). ${ }^{25-27}$ Very importantly, as effective communication helps build trust with the patient and ensures a proper transmission of information about the condition and treatment, ${ }^{25-28}$ it should be in place before the point at which the prescription is discussed.

PtC 3: barriers and facilitators of adherence of a specific patient to a specific prescription should be appropriately evaluated

Many different factors determining non-adherent behaviour in people with RMDs have been identified in many studies, covering various domains. The WHO generated a framework by which these factors were classified into five different domains: (i) patient-related, (ii) condition-related, (iii) therapy-related, (iv) the socioeconomic context and (v) the healthcare system. ${ }^{4}$ Our overview of SR of qualitative studies demonstrated that the list of barriers and facilitators is extensive, with many factors not being modifiable, and none of them being a sole predictor of non-adherence. ${ }^{19}$ 
Some factors change with time and can appear either to be a cause, or a consequence, of patient non-adherence (eg, depression can lead to less motivation to adhere, but it can also be a consequence of disease activity due to non-adherence). Barriers to adherence are considered complex and multi-faceted and non-adherence should never be perceived as patients' fault only (eg, the hospital pharmacy was not open the only day a patient could take off from work that month, or the patient's skills for self-injection are very limited). Based on the overview SR, ${ }^{19}$ we have produced a checklist of factors related to non-adherence, which could help HCPs to identify barriers to adherence at the individual level (see online supplemental table S1).

\section{PtC 4: patient education should be provided for people with RMDs as an integral part of standard care}

Most interventions with proven effectiveness in non-adherence include components of patient education, ${ }^{19}$ namely provision of knowledge or information, self-management programmes, cognitive behavioural interventions, mindfulness, stress management, individual consultations, sharing experiences among patients, motivational discussions, exercise counselling, lifestyle change interventions and self-help courses. With this $\mathrm{PtC}$, the task force wants to emphasise that 'all people with RMDs should have access to and be offered patient education throughout the course of their disease including as a minimum; at diagnosis, at pharmacological treatment change and when required by the patient's physical or psychological condition'. ${ }^{11}$

Of course, we cannot overwhelm the patient at diagnosis or treatment start with information, but studies agree in that patient education, either direct or supported via websites, ${ }^{29} 30$ brochures, $\mathrm{SMS}^{24} 25$ or e-health may reduce non-adherent behaviour. In order to be effective, education/information should include information about drugs, ${ }^{31} 32$ disease process, ${ }^{31} 32$ physical exercises, ${ }^{31}$ joint protection, ${ }^{31} 33$ pain control, ${ }^{313}$ coping strategies ${ }^{31}$ and lifestyle changes. ${ }^{32} 33$ Delivery formats can include verbally (face to face ${ }^{31}$ or by telephone ${ }^{34}$ ), written (as leaflets ${ }^{31}$ or using test messages ${ }^{29}{ }^{30}$ ) and visualised in charts ${ }^{35}$ (see online supplemental table S2).

\section{PtC 5: care should be tailored to patient preferences and goals to enhance adherence}

As already mentioned, the list of potential factors that can influence non-adherence to treatment is extensive and challenging to address. However, building a trust and a sound patient-HCPs relationship will prepare the scene for a responsible and blamefree framework that will reduce non-adherence in the long term. ${ }^{28}$ This tailored care has a maximum exponent during the SDM process, when options and patient preferences are the basis for an agreement to be treated and monitored. ${ }^{23}$

\section{PtC 6: adherence should be discussed regularly based on open} questions and particularly when disease is not well controlled

As adherence changes with time, the task force could not specify a best moment to assess non-adherence. Finally, we suggest that non-adherence should be assessed in a continuum. The task force discussed the opportunity to discuss non-adherence when the disease is not well controlled but specifically agreed to highlight the need for regularity. Regularly in this context would be, at a minimum, once per year.

Some experts within the TF argued to use validated measures of non-adherence, for instance, by the Medication Event Monitoring System, the level/dose ratio or the medication possession ratio. ${ }^{36}$ The reality is that the non-adherence construct has many grey areas and more than 200 ways to measure non-adherence to medication exist. ${ }^{37}$ The TF undertook an SR of instruments to screen non-adherence to medication and exercises in people with RMDs, including but not limited to validated questions, questionnaires, assessment and others (eg, pill counts, worn splint), without identifying a single measure that was clearly superior, neither for medication nor for exercises. ${ }^{19}$

In practical terms, whether non-adherence is a problem or not, it should be discussed through open conversation with the patient. The 'some people' approach may facilitate the generation of a safe space for more elaborated questions and answers ('We know it can be difficult, everybody has some problems, could you tell me what problems you encountered when taking your medication?' or 'Could you show me how you actually wear the splint? How do you actually perform the exercises?'). ${ }^{28}$ Other forms of screening, like questionnaires, looking at pharmacy indicators, drug levels or wearables can be used, always tailored to patient preferences and goals to reduce non-adherence, but a single measure without the open discussion is not recommended.

\section{PtC 7: the HCP should explore which factors might negatively} influence adherence, including opportunity (eg, availability or cost), capability (eg, memory problems) or motivation (eg, concerns)

This PtC adds a method or systematic approach to explore barriers to adherent behaviour. We used the Capability, Opportunity and Motivation model of Behaviour (COM-B) as framework to explore patient's drivers for non-adherence and as instrument to identify possible targets for reducing nonadherence. ${ }^{38}$ The model acknowledges that behaviour is part of an interacting, dynamic system involving these components to determine a person's non-adherence. The problems that are easiest to address are those of practical nature-for example, unavailability at local pharmacy, pharmacy open hours, interference with occupation - and thus they should be the first ones explored. Next would be knowledge or capability related, for example, the patient does not really understand the duration of treatment, what to do in case of missing dose, or how to inject. These can be explored by asking the patient, with some level of detail, how she or he actually takes the medication or, in the case of exercises, how he or she performs the exercises. Regardless of problems in these previous areas, the motivation to adhere, or intention, has to be explored. This is usually explored in terms of needs and concerns. ${ }^{1}$

PtC 8: together with the patient, the HCP should tailor the approach to overcome individual barriers to adherence, for example, simplifying the regimen, using reminders, providing education, discussing the patient's beliefs on treatments

Based on the reasons for non-adherence, the solutions must be tailored to tackle the specific problems. There is not general recipe or 'one size fits all' as how to do this and solutions to tackle non-adherence are plenty. The two steps of a tailored approach would be (1) to identify the reasons of non-adherence, including the assessment of problems, as well as low health literacy or skills, and (2) to focus on the specific problems, modulating the intervention to the individual (eg, a patient clearly needs a reminder but does not have a smartphone, we should recommend the use of pill boxes with timers). Some randomised controlled trials that specifically named their strategies as 'tailored' showed positive results. ${ }^{32} 34{ }^{39-41}$ Many include revising treatment schemes to make them as convenient and easy to follow as possible.

Besides patient education strategies, already highlighted, there are simple things that can be done, like advising on cueing 
behaviours (eg, pairing medication taking with an established behaviour, such as brushing teeth), ${ }^{42}$ monitoring (eg, using a calendar or a diary to track medication taking), ${ }^{42}$ including the family and close ones in the intervention, ${ }^{42}$ review plans/ strategies and give feedback (including positive reinforcement) and answers. ${ }^{42}$ Individualising the prescription and regimen according to the preferences and goals of the patient has been proven effective. ${ }^{43}$ In the case of exercises, it has been suggested to split treatment visits, ${ }^{44}$ increase proxy efficacy (ie, patients' confidence in the therapist's ability to function effectively on his/her behalf; this can be done by showing that the therapist competency really aids achieving goals) ${ }^{45}$ and discuss barriers and facilitators of exercises with the patient, ${ }^{46}$ encouraging him/ her to plan own treatment regimens, discussing intentions and helping recasting unrealistic plans, ${ }^{46}$ individualising physical activity advice, ${ }^{40}$ tailor graded exercise programme, ${ }^{47}$ training in the proper execution of physical exercises ${ }^{46}$ and providing visual media of the prescribed exercises and explanatory written information. ${ }^{48}$ A list of practical things is shown in the online supplemental table S2.

\section{PtC 9: when specific expertise or interventions for adherence are} needed, they should be made available to patients

Some interventions, especially those related to avoid intentional non-adherence may need specialised skills, such as motivational interviewing. ${ }^{24}$ If the care team includes a psychologist, she or he should be ideally involved, either in the management of the individual patients or as trainer or consultant for the team members. Other skills, such as being able to deliver effective communication, are insufficiently incorporated in most teams.

\section{DISCUSSION}

The topic of non-adherence is of utmost importance and yet not adequately addressed in rheumatology. Non-adherence both results in poorer outcomes and also in increased resource use and medical costs. ${ }^{49}$ Non-adherence varies across RMDs, being critical in gout, ${ }^{50} 51$ and osteoporosis, ${ }^{52}$ but also in rheumatoid arthritis, where it even vary across medication. ${ }^{49}$ The work of this multidisciplinary taskforce has highlighted both the complexity and a possible practical approach to non-adherence to prescribed treatments in RMDs.

The WHO definition of adherence does not easily translate into operational terms, as it relates to a behaviour. ${ }^{16}$ Nonadherence can take many different forms: the patient could actually be overdosing the medication, the exercises or the use of devices, or not using it as prescribed (eg, misapplying medication or exercises, or using splints ineffectively). The complexity and difficulty of identifying factors accurately and predicting medication non-adherence has led to the development of cognitive models to better explain this complex phenomenon. ${ }^{1253}$ These models take into account areas such as illness beliefs, expectations, barriers and intentions, and have become the basis of measurement instruments. ${ }^{36}$ In practical terms, and in the context of a frank discussion, we should ask the patient about his or her beliefs and concerns about treatments, as these are universally present in patients on long-term treatments. ${ }^{54}$ In this line, Foot et al found a moderate effect of pharmacological treatment necessity in rheumatoid arthritis and systemic sclerosis-both being the RMDs with largest 'need' belief of treatment-and lowest in osteoporosis, while they found a strong negative association between concerns about adverse effects of taking medicines and non-adherence in rheumatoid arthritis and in osteoporosis and moderate in systemic sclerosis. ${ }^{55}$ Patients will always weigh up necessity and concern, and therefore we have a 'window of opportunity' for patient education and counselling.

Patient-centeredness and shared decisions are key elements in relation to (non-)adherence. If perspectives and preferences of patients are not adequately taken into account in medical decisions, non-adherence might get a paternalistic connation. Instead the HCP 'telling' the patient what to do and the patient needing to follow this advice, a common patient-centric perspective should be established, and patients should be encouraged to take active role in the subsequent decision. However, we acknowledge, that it is not possible for HCPs to assess or be certain that a patient has agreed to the proposed treatment plan, especially because some patients may give socially desirable answers (for fear of disappointing the HCPs). Therefore, adherence should be discussed regularly, and the patients must be given assurance that they can be honest, because it is their right not to take the treatment as prescribed. However, the patients should also be encouraged to tell the HCPs about not taking the treatment as only with mutual trust, optimal can take place.

The aim of our taskforce was to be as practical as possible. Other groups have already issued recommendations to reduce non-adherence in RMDs, ${ }^{56}$ but they dealt specifically with medication and rheumatoid arthritis, and we wanted to be broader. A practical local initiative designed a model for prescription to tackle non-adherence, ${ }^{57}$ and in a very practical paper, Rashid et al synthesise the implications of qualitative research in the field of non-adherence into the following 11 statements: (1) individualise care plan, (2) address practical barriers for the individual, (3) adopt a patient-centred approach, (4) increase HCPs involvement, (5) ensure long-term follow-up, (6) promote self-management, (7) increase family or carer involvement, (8) improve patient education, (9) address system barriers, (10) increase access to non-prescribing HCPs and (11) improve staff training. ${ }^{58}$ Many of these suggestions reflect our views and conclusions.

Exercises are a pillar in the treatment of most RMDs and nonadherence may be even larger than to medication. We realise that both the definition of adherence and most of the literature deal with non-adherence to medication, and thus will not entirely apply to exercises or to other prescribed treatments, such as use of splints. However, due to the generic nature of the PtCs, we assume that our results can equally be applied to any treatment the patient is receiving and thus will cover exercises as well.

Two aspects differ from the initial plans of this taskforce. First, prevention was not initially included as an objective of the taskforce; however, it soon became clear that prevention is the ultimate solution to avoid non-adherence, and strategies to achieve it will probably overlap with those of an optimal SDM process. Second, in the proposal approved by the EULAR executive, non-adherence to visits and to diet, and not only to medication or exercises, were initially included. However, the task force thought that visits and diet were too complex to measure, and would overlap with other initiatives, and left them out of the scope and the research agenda.

Finally, regarding the implementation of these PtCs, the task force wanted to stress, on one hand the need to adopt a truly patient-centred approach and, on the other, the need to make system changes. Several PtCs involve the patient as main stakeholder in the issue of non-adherence. Nothing can be done in terms of non-adherence without the help of the one who agrees, or not, to follow the treatment or exercises as prescribed. To engage him or her, we will need to attain basic effective communication skills and make SDM a reality. The consequences will be a better outcome and higher odds of adequate self-management. 
Similarly, if we do not evaluate periodically non-adherence at an organisational level, and address system barriers, many of which are modifiable, including improved staff training, we will not be able to make effective changes, or measure the impact of implemented strategies. There is enough information to support better use of existing resources and treatments, this include reducing non-adherence.

In summary, these PtCs can help HCPs to support people with RMDs to adhere to the agreed treatment plan, the basic scheme being minimise non-adherence by bonding with the patient and building trust, and by overcoming structural barriers, assess in a blame-free environment and tailor the solution to the problem.

\section{Author affiliations}

${ }^{1}$ Section for Outcomes Research, Medical University of Vienna, Wien, Austria

${ }^{2}$ Ludwig Boltzmann Institute for Arthritis and Rehabilitation, Wien, Austria

${ }^{3}$ Department of Rheumatology, Medical University of Vienna, Wien, Austria

${ }^{4}$ Rheumatology, UMC Utrecht, Utrecht, The Netherlands

${ }^{5}$ Deutsche Rheuma-Liga Bundesverband e.V, Bonn, Germany

${ }^{6}$ Rehabilitation, Physical Medicine and Rheumatology, University of Medicine and Pharmacy Victor Babes Timisoara, Timisoara, Romania

7 Department of Nursing and Midwifery, University of the West of England, Bristol, UK

${ }^{8}$ Academic Rheumatology, Bristol Royal Infirmary, Bristol, UK

${ }^{9}$ Department of Child and Adolescent Psychiatry/Psychology, Erasmus MC University Medical Center, Rotterdam, The Netherlands

${ }^{10}$ Institut Pierre Louis d'Epidémiologie et de Santé Publique, INSERM, Sorbonne Universite, Paris, France

${ }^{11}$ APHP, Rheumatology Department, Hopital Universitaire Pitie Salpetriere, Paris, France

${ }^{12}$ Scienze Cliniche e Biologiche, Università degli Studi di Torino, Torino, Italy

${ }^{13}$ Rheumatology Department, Centro Hospitalar e Universitário de Coimbra, Health Sciences Research Unit: Nursing, Coimbra, Portugal

${ }^{14}$ Division of Rheumatology and Research, Diakonhjemmet Hospital, Oslo, Norway

${ }^{15}$ Mifrakim Tz'eirim, Haifa, Israel

${ }^{16}$ Pharmacy, Sint Maartenskliniek, Nijmegen, The Netherlands

${ }^{17}$ Department of Pharmacy, Radboud Univiersity Medical Center, Nijmegen, The Netherlands

${ }^{18}$ Hospital Pharmacy, Diakonhjemmet Hospital Pharmacy, Oslo, Norway

${ }^{19}$ School of Pharmacy, University of Oslo, Oslo, Norway

${ }^{20}$ Department Psychology, Health and Technology, University of Twente, Enschede, The Netherlands

${ }^{21}$ Department of Rheumatology, Aarhus University Hospital, Aarhus, Denmark

${ }^{22}$ Department of Clinical Medicine, Aarhus University, Aarhus, Denmark

${ }^{23}$ Instituto de Salud Musculoesquelética (INMUSC), Madrid, Spain

Twitter Fernando Estévez-López @FerEstevezLope1 and Loreto Carmona @ carmona loreto

Acknowledgements We thank Conni Skrubbeltrang (Chief Librarian at Aalborg Universitetshospital, Denmark) and José Bernardo Negrón Torres (Instituto de Investigación Social y Sanitaria, Puerto Rico) for their contribution to the systematic literature search.

Contributors All listed authors of the manuscript are the members of the EULAR assigned task force dedicated to the development of points to consider for adherence to treatment in people with rheumatic and musculoskeletal diseases. All authors contributed to the formulation of the points to consider. In details, the steering committee of the task force (LC, VR and AdT) defined the research questions for the systematic literature reviews. The search was performed by a research Liberian (CS), under supervision of the methodologist (AdT). JBNT, AdT and LC selected the studies, VR assessed risk of bias of all eligible studies. VR, LC and JBNT extracted the data, and synthesised the results. LC and AdT reviewed processes, excluded articles and tailored the synthesis reports. The manuscript was drafted by VR and revised by TAS, AdT and LC. All other authors participated in the taskforce meetings, suggested and agreed upon the research questions, read the report prior to the manuscript, discussed results and made contributions to the text. All authors approved the final version of the manuscript.

Funding This project was funded by EULAR (project number HPR037)

Competing interests TAS has received grant/research support from AbbVie, and Roche, has been consultant for AbbVie, Sanofi Genzyme, and has been paid speaker for AbbVie, Roche and Sanofi. DA has received grant/research support from AbbVie, Amgen, Celgene, Lilly, Medac, Merck, Novartis, Pfizer, Roche, Sandoz, Sanofi Genzyme and UCB, has been consultant for AbbVie, Amgen, Celgene, Lilly, Medac, Merck, Novartis, Pfizer, Roche, Sandoz, Sanofi Genzyme and UCB and has been paid speaker for AbbVie, Amgen, Celgene, Lilly, Medac, Merck, Novartis, Pfizer, Roche, Sandoz, Sanofi Genzyme and UCB. JWJB has received grant/research support from
Roche, and has been paid speaker for Roche and Lilly. RGD has been paid speaker for MSD, AbbVie, Novartis, Roche, Pfizer, Myllan and Sandoz. ED has received grant/research support from Independent Learning, Pfizer, combined funding for a research fellow from Celgene, Abbvie and Novartis, and has been paid instructor for Novartis to deliver training to nurses. LG has received grant/research support from Lilly, Pfizer and Sandoz, and has been consultant for AbbVie, Amgen, Biogen, BMS, Celgene, Janssen, Lilly, MSD, Novartis, Pfizer, Sandoz, Sanofi-Aventis and UCB Pharma. BJFvdB has been paid speaker for MSD, Abbvie and Biogen. MV has been paid speaker for Pfizer. AdT has received grants from Novartis, and has been paid speaker for Lilly and Pfizer. LC has received grant/research support through her institute from Novartis, Pfizer, MSD, Roche, Sanofi Aventis, AbbVie and Gebro Pharma.

\section{Patient consent for publication Not required.}

Provenance and peer review Not commissioned; externally peer reviewed.

Supplemental material This content has been supplied by the author(s). It has not been vetted by BMJ Publishing Group Limited (BMJ) and may not have been peer-reviewed. Any opinions or recommendations discussed are solely those of the author(s) and are not endorsed by BMJ. BMJ disclaims all liability and responsibility arising from any reliance placed on the content. Where the content includes any translated material, BMJ does not warrant the accuracy and reliability of the translations (including but not limited to local regulations, clinical guidelines, terminology, drug names and drug dosages), and is not responsible for any error and/or omissions arising from translation and adaptation or otherwise.

\section{ORCID iDs}

Valentin Ritschl http://orcid.org/0000-0001-8763-8215

Tanja A Stamm http://orcid.org/0000-0003-3073-7284

Daniel Aletaha http://orcid.org/0000-0003-2108-0030

Johannes W J Bijlsma http://orcid.org/0000-0002-0128-8451

Peter Böhm http://orcid.org/0000-0002-7186-1997

Razvan Gabriel Dragoi http://orcid.org/0000-0002-3991-7583

Emma Dures http://orcid.org/0000-0002-6674-8607

Fernando Estévez-López http://orcid.org/0000-0003-2960-4142

Laure Gossec http://orcid.org/0000-0002-4528-310X

Annamaria lagnocco http://orcid.org/0000-0001-5592-724X

Andrea Marques http://orcid.org/0000-0002-2026-9926

Bart J F van den Bemt http://orcid.org/0000-0002-8560-9514

Kirsten Viktil http://orcid.org/0000-0003-2361-5107

Marieke Voshaar http://orcid.org/0000-0002-4161-0126

Annette de Thurah http://orcid.org/0000-0003-0103-4328

Loreto Carmona http://orcid.org/0000-0002-4401-2551

\section{REFERENCES}

1 van den Bemt BJF, Zwikker HE, van den Ende CHM. Medication adherence in patients with rheumatoid arthritis: a critical appraisal of the existing literature. Expert Rev Clin Immunol 2012;8:337-51.

2 de Achaval S, Suarez-Almazor ME. Treatment adherence to disease-modifying antirheumatic drugs in patients with rheumatoid arthritis and systemic lupus erythematosus. Int I Clin Rheumtol 2010;5:313-26.

3 DiMatteo MR. Variations in patients' adherence to medical recommendations: a quantitative review of 50 years of research. Med Care 2004;42:200-9.

4 World Health Organization. Adherence to long-term therapies: evidence for action. World Health Organization, 2003.

5 van Breukelen-van der Stoep DF, Zijlmans J, van Zeben D, et al. Adherence to cardiovascular prevention strategies in patients with rheumatoid arthritis. Scand J Rheumatol 2015;44:443-8.

6 Nordgren B, Fridén C, Demmelmaier I, et al. An outsourced health-enhancing physical activity programme for people with rheumatoid arthritis: exploration of adherence and response. Rheumatology 2015;54:1065-73.

7 Bruce IN, Gladman DD, Urowitz MB. Factors associated with refractory renal disease in patients with systemic lupus erythematosus: the role of patient nonadherence. Arthritis Care Res 2000;13:406-8.

8 Smolen JS, Landewé R, Breedveld FC, et al. EULAR recommendations for the management of rheumatoid arthritis with synthetic and biological disease-modifying antirheumatic drugs: 2013 update. Ann Rheum Dis 2014;73:492-509.

9 Fernandes L, Hagen KB, Bijlsma JWJ, et al. EULAR recommendations for the nonpharmacological core management of hip and knee osteoarthritis. Ann Rheum Dis 2013;72:1125-35.

10 Bech B, Primdahl J, van Tubergen A, et al. 2018 update of the EULAR recommendations for the role of the nurse in the management of chronic inflammatory arthritis. Ann Rheum Dis 2020;79:61-8.

11 Zangi HA, Ndosi M, Adams J, et al. EULAR recommendations for patient education for people with inflammatory arthritis. Ann Rheum Dis 2015;74:954-62.

12 Voshaar M, Vriezekolk J, van Dulmen S, et al. Barriers and facilitators to diseasemodifying antirheumatic drug use in patients with inflammatory rheumatic diseases: a qualitative theory-based study. BMC Musculoskelet Disord 2016;17:442. 
13 Brandstetter S, Hertig S, Loss J, et al. 'The lesser of two evils...' - views of persons with rheumatoid arthritis on medication adherence: a qualitative study. Psychol Health 2016;31:675-92.

14 De Civita M, Feldman DE, Meshefedjian GA, et al. Caregiver recall of treatment recommendations in juvenile idiopathic arthritis. Arthritis Rheum 2007;57:219-25.

15 Allen KD, Warzak WJ. The problem of parental nonadherence in clinical behavior analysis: effective treatment is not enough. J Appl Behav Anal 2000;33:373-91.

16 World Health Organisation. Global strategy on diet, physical activity and health, 2018 Available: https://www.who.int/dietphysicalactivity/pa/en/ [Accessed 29 Dec 2018].

17 Rausch Osthoff A-K, Niedermann K, Braun J, et al. 2018 EULAR recommendations for physical activity in people with inflammatory arthritis and osteoarthritis. Ann Rheum Dis 2018;77:1251-60.

18 van der Heijde D, Aletaha D, Carmona L, et al. 2014 update of the EULAR standardised operating procedures for EULAR-endorsed recommendations. Ann Rheum Dis 2015;74:8-13.

19 Ritschl V, Stamm TA, Aletaha D, et al. Prevention, screening, assessing and managing of non-adherent behaviour in people with rheumatic and musculoskeletal diseases: systematic reviews Informing the 2020 EULAR points to consider. RMD Open 2020:6:e001432.

20 Higgins JPT, Altman DG, Gøtzsche PC, et al. The Cochrane collaboration's tool for assessing risk of bias in randomised trials. BMJ 2011;343:d5928.

21 Whiting PF, Rutjes AWS, Westwood ME, et al. QUADAS-2: a revised tool for the quality assessment of diagnostic accuracy studies. Ann Intern Med 2011;155:529-36.

22 Centre for Evidence-Based Medicine. OCEBM levels of evidence, 2016. Available: https://www.cebm.net/2016/05/ocebm-levels-of-evidence/

23 Elwyn G, Laitner S, Coulter A, et al. Implementing shared decision making in the NHS. BMJ 2010;341:c5146.

24 Nieuwlaat R, Wilczynski N, Navarro T, et al. Interventions for enhancing medication adherence. Cochrane Database Syst Rev 2014:Cd000011.

25 Zolnierek KBH, Dimatteo MR. Physician communication and patient adherence to treatment: a meta-analysis. Med Care 2009;47:826-34.

26 Bukstein DA. Patient adherence and effective communication. Ann Allergy Asthma Immunol 2016;117:613-9.

27 Zulman DM, Haverfield MC, Shaw JG, et al. Practices to foster physician presence and connection with patients in the clinical encounter. JAMA 2020;323:70-81.

28 Brown MT, Bussell J, Dutta $\mathrm{S}$, et al. Medication adherence: truth and consequences. Am J Med Sci 2016:351:387-99.

29 Balato N, Megna M, Di Costanzo L, et al. Educational and motivational support service: a pilot study for mobile-phone-based interventions in patients with psoriasis. Br J Dermatol 2013;168:201-5.

30 Ting TV, Kudalkar D, Nelson S, et al. Usefulness of cellular text messaging for improving adherence among adolescents and young adults with systemic lupus erythematosus. J Rheumatol 2012;39:174-9.

31 Hill J, Bird H, Johnson S. Effect of patient education on adherence to drug treatment for rheumatoid arthritis: a randomised controlled trial. Ann Rheum Dis 2001;60:869-75.

32 Ganachari MS, Almas SA. Evaluation of clinical pharmacist mediated education and counselling of systemic lupus erythematosus patients in tertiary care hospital. Indian $J$ Rheumatol 2012;7:7-12

33 El Miedany Y, El Gaafary M, El Arousy N, et al. Arthritis education: the integration of patient-reported outcome measures and patient self-management. Clin Exp Rheumatol 2012;30:899-904.

34 Clifford S, Barber N, Elliott R, et al. Patient-centred advice is effective in improving adherence to medicines. Pharm World Sci 2006;28:165-70.

35 El Miedany Y, El Gaafary M, Palmer D. Assessment of the utility of visual feedback in the treatment of early rheumatoid arthritis patients: a pilot study. Rheumatol Int 2012:32:3061-8

36 Forbes CA, Deshpande S, Sorio-Vilela F, et al. A systematic literature review comparing methods for the measurement of patient persistence and adherence. Curr Med Res Opin 2018;34:1613-25.

37 Kelly A, Sumpton D, O'Sullivan C, et al. Scope and consistency of adherence related outcomes in randomized controlled trials of interventions for improving medication adherence. Arthritis Rheum 2017.
38 Jackson C, Eliasson L, Barber N, et al. Applying COM-B to medication adherence: a suggested framework for research and interventions. Eur Health Psychol 2014;16:7-17

39 Galo JS, Mehat P, Rai SK, et al. What are the effects of medication adherence interventions in rheumatic diseases: a systematic review. Ann Rheum Dis 2016;75:667-73.

40 Halbert J, Crotty M, Weller D, et al. Primary care-based physical activity programs: effectiveness in sedentary older patients with osteoarthritis symptoms. Arthritis Rheum 2001;45:228-34.

41 Ezzat AM, MacPherson K, Leese J, et al. The effects of interventions to increase exercise adherence in people with arthritis: a systematic review. Musculoskeletal Care 2015;13:1-18

42 Rapoff MA, Belmont J, Lindsley C, et al. Prevention of nonadherence to nonsteroidal anti-inflammatory medications for newly diagnosed patients with juvenile rheumatoid arthritis. Health Psychol 2002;21:620-3.

43 Evers AWM, Kraaimaat FW, van Riel PLCM, et al. Tailored cognitive-behavioral therapy in early rheumatoid arthritis for patients at risk: a randomized controlled trial. Pain 2002;100:141-53.

44 Ravaud P, Flipo R-M, Boutron I, et al. Artist (osteoarthritis intervention standardized) study of standardised consultation versus usual care for patients with osteoarthritis of the knee in primary care in France: pragmatic randomised controlled trial. BMJ 2009;338:b421.

45 Vong SK, Cheing GL, Chan F, et al. Motivational enhancement therapy in addition to physical therapy improves motivational factors and treatment outcomes in people with low back pain: a randomized controlled trial. Arch Phys Med Rehabil 2011:92:176-83.

46 Brus HL, van de Laar MA, Taal E, et al. Effects of patient education on compliance with basic treatment regimens and health in recent onset active rheumatoid arthritis. Ann Rheum Dis 1998;57:146-51.

47 Pisters MF, Veenhof $\mathrm{C}$, de Bakker $\mathrm{DH}$, et al. Behavioural graded activity results in better exercise adherence and more physical activity than usual care in people with osteoarthritis: a cluster-randomised trial. J Physiother 2010;56:41-7.

48 Cifcili $\mathrm{S}, \mathrm{Akman} \mathrm{M}$, et al. How can we improve adherence to exercise programs in patients with osteoarthritis?: a randomized controlled trial. Turk J Geriatr 2012;15.

49 Fautrel B, Balsa A, Van Riel P, et al. Influence of route of administration/ drug formulation and other factors on adherence to treatment in rheumatoid arthritis (pain related) and dyslipidemia (non-pain related). Curr Med Res Opin 2017;33:1231-46

50 Scheepers LEJM, van Onna M, Stehouwer CDA, et al. Medication adherence among patients with gout: a systematic review and meta-analysis. Semin Arthritis Rheum 2018;47:689-702.

51 Yin R, Li L, Zhang G, et al. Rate of adherence to urate-lowering therapy among patients with gout: a systematic review and meta-analysis. BMJ Open 2018;8:e017542.

52 Bianchi ML, Duca P, Vai S, et al. Improving adherence to and persistence with oral therapy of osteoporosis. Osteoporos Int 2015;26:1629-38.

53 Horne R. Treatment perceptions and self-regulation. In: Cameron LLH, ed. The selfregulation of health and illness behaviour. Routledge, 2003: 138-53.

54 van den Bemt BJF, van Lankveld WGJM. How can we improve adherence to therapy by patients with rheumatoid arthritis? Nat Clin Pract Rheumatol 2007:3:681.

55 Foot H, La Caze A, Gujral G, et al. The necessity-concerns framework predicts adherence to medication in multiple illness conditions: a meta-analysis. Patient Educ Couns 2016:99:706-17.

56 Gossec L, Molto A, Romand X, et al. Recommendations for the assessment and optimization of adherence to disease-modifying drugs in chronic inflammatory rheumatic diseases: a process based on literature reviews and expert consensus. Joint Bone Spine 2019;86:13-19.

57 González-Bueno J, Calvo-Cidoncha E, Sevilla-Sánchez D, et al. Patient-centered prescription model to improve therapeutic adherence in patients with multimorbidity. Farm Hosp 2018;42:128-34.

58 Rashid MA, Llanwarne N, Heyns N, et al. What are the implications for practice that arise from studies of medication taking? A systematic review of qualitative research PLoS One 2018:13:e0195076. 